\title{
Micromanaging dopamine neurons
}

DOI:

10.1038/nrn2246

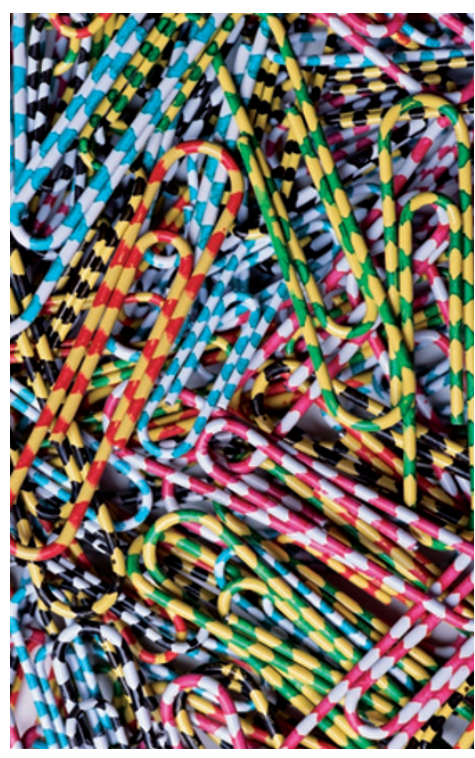

As our understanding of the finetuning of neuronal gene expression has grown, microRNAs (miRNAs) have emerged as key posttranscriptional regulators. These small, non-coding RNAs prevent the translation of target mRNAs, thereby helping to determine a cell's eventual protein make-up. Abeliovich and colleagues now demonstrate the importance of miRNA activity for midbrain dopamine neuron function.

There is keen interest in understanding the genetic programmes that drive the development and maintenance of dopamine neurons, owing to the importance of these cells in the pathology of Parkinson's disease (PD). The authors set out to determine the role of miRNAs in this process. They began by creating a murine embryonic stem cell (ES) line in which Dicer, an enzyme that is crucial for miRNA production, was deleted. They were unable to generate dopamine neurons from these cells, suggesting that miRNAs have a role in dopamine neuron differentiation and/or maintenance.

To examine the importance of miRNA activity in vivo, the authors generated transgenic mice in which Dicer was specifically deleted in postmitotic midbrain dopamine neurons. As the mice aged, they progressively lost midbrain dopamine neurons and displayed reduced locomotor activity - a phenotype that is reminiscent of the symptoms of patients with PD, indicating that altered miRNA activity might contribute to PD pathology.

To determine which of the hundreds of miRNAs that are expressed in the brain are involved in maintaining midbrain dopamine neurons, the authors compared the expression of 224 miRNAs in brain samples from a small number of PD patients with controls. One particular miRNA, miR-133b, was highly expressed in the midbrain of controls, but was reduced in the midbrain of PD patients.

Lower levels of miR-133b were also found in the midbrain of two rodent models of dopamine deficiency. One of these, the Aphakia mouse, carries a mutation in the gene that encodes PITX3, suggesting that miR-133b expression might be regulated by this protein. Indeed, the authors found that PITX3 overexpression increased levels of miR-133b. They went on to show that PITX3 mRNA is itself a potential target for miR-133b. PITX3 protein levels were increased in rat midbrain cells in which miR-133b was suppressed using antisense oligodeoxynucleotides. This suggests that a negative-feedback circuit exists in which PITX3 induces miR-133b expression and then is itself downregulated by miR-133b activity.

Somewhat surprisingly, the authors found that overexpression of miR-133b in ES or primary midbrain cultures reduced the expression of markers of dopamine neuron differentiation and reduced dopamine release in response to a depolarizing stimulus. Furthermore, suppressing miR-133b activity increased the expression of dopaminergic markers and potentiated dopamine release. These results indicate that further work will be needed to understand the precise role of $\mathrm{miR}-133 \mathrm{~b}$ in dopamine differentiation and function.

Although important questions remain to be answered regarding the full repertoire of miR-133b activity, this study provides evidence for the importance of miRNAs in the maintenance of dopamine neurons, and suggests that researchers should continue to look closely for possible links between disruptions in this form of post-transcriptional regulation and disease pathology.

Katherine Whalley

ORIGINAL RESEARCH PAPER Kim, J. et al. A microRNA feedback circuit in midbrain dopamine neurons. Science 317, 1220-1224 (2007) 